\title{
AN EXTENSION OF MILLER'S VERSION OF THE DE RHAM THEOREM WITH ANY COEFFICIENTS
}

\author{
ANTONIO GARVÍN, LUIS LECHUGA, \\ ANiCETO MURILlO, VICENTE MUÑOZ and ANTONiO VIRUEL \\ Departamento de Algebra, Geometría y Topología, Universidad de Málaga \\ Ap. 59, 29080 Málaga, Spain \\ E-mail: aniceto@agt.cie.uma.es
}

\begin{abstract}
In this paper we present an approximation to the de Rham theorem for simplicial sets with any coefficients based, using simplicial techniques, on Poincaré's lemma and $q$-extendability.
\end{abstract}

1. Introduction. The commutative cochain problem consists in finding, for a given topological space $X$, a functorial commutative differential graded algebra $A(X)$ which is "extendable" (i.e., for a subspace $Y \subset X, A(X) \rightarrow A(Y)$ is surjective) and whose cohomology algebra yields the usual singular cohomology of the space. Over $\mathbb{R}$ a solution was given by Thom in 1957 (unpublished) and, later on, D. Quillen [7] solved this problem when the coefficient field is $\mathbb{Q}$. D. Sullivan [8] found a more geometrical solution when defining the polynomial forms on a topological space. Almost at the same time, R. Swan [9] gave a similar construction, also over $\mathbb{Q}$, in which already the facts of "extendability" and "Poincaré's lemma" were crucial for his purposes.

However it is well known that, over the integers, the commutative cochain problem cannot be solved (see for example [2]), cohomology operations being obstructions to achieve such a solution. Nevertheless, it is of natural interest to approximate the singular cohomology of a space with any coefficients using, when possible, objects analogous to Swan and Sullivan's polynomial differential forms. This was indeed done by E. Y. Miller [6] for a finite simplicial complex: he defined the differential forms of length $q$ over a finite simplicial complex $A^{*, q}(X)$ (which is no longer an algebra but a module over the given coefficient ring) and showed that the integration $\int: A^{*, q}(X) \rightarrow C^{*}(X)$ induces

1991 Mathematics Subject Classification: 18G30, 55P60, 58A12.

Key words and phrases: de Rham theorem, commutative cochain problem, simplicial set.

Partially supported by a DGICYT grant (PB94-1485).

The paper is in final form and no version of it will be published elsewhere. 
an isomorphism in cohomology up to degree $q$. This was done following the original and standard sheaf theoretic proof of the de Rham theorem.

More generally, over any simplicial set, the only reference we are aware of is the one of H. Cartan [1] where in $\S 7$ he sketched an analogous result given, according to Cartan, by Grothendieck at a conference in the IHES.

In this paper we present a generalization of Miller's result over any simplicial set. We use simplicial techniques and following the approach of $[3 ; \S 10]$ or [4], we show that in order to have a de Rham theorem up to a given degree $q$ over any coefficients is enough to have $q$-extendability (proposition 3.2) and Poincaré's lemma (proposition 3.1).

In the next section we give the basic properties we shall need from the simplicial setting. In $\S 3$ we prove that the simplicial differential graded module of polynomial forms of fixed length $q$ is " $q$-extendable" and satisfies Poincaré's lemma. Finally, in section 4 we state the main results.

2. A short trip to the simplicial world. Most of the contents in this section are well known results and basic facts about simplicial objects. Hence we shall not give proofs for some of the assertions and we refer to [3] (where a very short and nice exposition can be found), [4] or [5] for complete proofs and details. From now on our coefficient ring $R$ is commutative and with unity.

A simplicial object $K$ with values in a category $\mathcal{C}$ is a sequence of objects in $\mathcal{C}$, $\left\{K_{n}\right\}_{n \geq 0}$, together with morphisms $\partial_{i}: K_{n+1} \rightarrow K_{n}, 0 \leq i \leq n+1$, and $s_{j}: K_{n} \rightarrow$ $K_{n+1}, 0 \leq j \leq n$, called respectively face and degeneracy operators, satisfying the usual relations, i.e.,

$$
\begin{aligned}
\partial_{i} \partial_{j} & =\partial_{j-1} \partial_{i}, \quad i>j, \\
\partial_{i} s_{j} & = \begin{cases}s_{j-1} \partial_{i}, & i<j, \\
i d, & i=j, j+1, \\
s_{j} \partial_{i-1}, & i>j+1,\end{cases} \\
s_{i} s_{j} & =s_{j+1} s_{i}, \quad i \leq j .
\end{aligned}
$$

Given $K$ and $L$ two simplicial objects in $\mathcal{C}$, a simplicial morphism $\varphi: K \rightarrow L$ is a sequence of morphisms of $\mathcal{C}, \varphi_{n}: K_{n} \rightarrow L_{n}, n \geq 0$, commuting with the face and degeneracy operators. Hence, a simplicial set is nothing but a simplicial object in the category of sets. In this particular context, given a simplicial set $K$ we shall pay special attention to its skeletons: for any $m \geq 0$ the $m$-skeleton of $K, K(m)$, is the subsimplicial set of $K$ defined by

$$
K(m)_{n}= \begin{cases}K_{n}, & n \leq m, \\ s_{i} \sigma, & 1 \leq i \leq n-1, \sigma \in K(m)_{n-1}, \quad n>m .\end{cases}
$$

We say that a simplicial set $K$ has dimension $m$ if $K=K(m)$. The degenerate $n$-simplices are, by definition, the image of the degeneracy operators: $s_{i} \sigma, 0 \leq i \leq n-1, \sigma \in K_{n-1}$.

A very useful example is, for a given integer $k \geq 0$, the simplicial set $\Delta[k]$ which can be thought of as the subsimplicial set of the singular simplices on $\Delta^{k}$ formed by the linear simplices, i.e.,

$$
\Delta[k]_{n}=\left\{\sigma=<e_{i_{0}}, \ldots, e_{i_{n}}>, \quad 0 \leq i_{0} \leq \ldots \leq i_{n} \leq k\right\}
$$


where $<e_{i_{0}}, \ldots, e_{i_{n}}>$ denotes the linear simplex $\Delta^{n} \rightarrow \Delta^{k}$ which sends the vertex $e_{j}$ to $e_{i_{j}}, 0 \leq j \leq n$. The face and degeneracy operators are induced respectively by the inclusions and the projections:

$$
\begin{aligned}
& \lambda_{i}=<e_{0}, \ldots, \hat{e}_{i}, \ldots, e_{n+1}>: \Delta^{n} \longrightarrow \Delta^{n+1}, \\
& \rho_{j}=<e_{0}, \ldots, e_{j}, e_{j}, \ldots, e_{n}>: \Delta^{n+1} \longrightarrow \Delta^{n} .
\end{aligned}
$$

Observe that the dimension of $\Delta[k]$ is precisely $k$ and that there is just one non-degenerate $k$-simplex in $\Delta[k]$, the identity $1_{\Delta^{k}}$.

Given $K$ a simplicial set and $A$ a simplicial cochain complex or simplicial differential graded $R$-module (sdgm in what follows), define $A(K)$ the differential graded module of forms on $K$ with coefficients in $A$ by

$$
A(K)=\oplus_{p \geq 0} A^{p}(K)
$$

where $A^{p}(K)$ are the simplicial set morphisms from $K$ into the simplicial set $A^{p}=$ $\left\{A_{n}^{p}\right\}_{n \geq 0}$. That is to say, an element $\Phi$ of degree $p$ of $A(K)$ is a collection $\left\{\Phi_{\sigma}\right\}_{\sigma \in K}$ in which $\Phi_{\sigma} \in A_{n}^{p}$ if $\sigma \in K_{n}$ and $\Phi_{\partial_{i} \sigma}=\partial_{i} \Phi_{\sigma}, \Phi_{s_{j} \sigma}=s_{j} \Phi_{\sigma}$, for any $i, j$. The differential is given by $d\left\{\Phi_{\sigma}\right\}=\left\{d \Phi_{\sigma}\right\}$. Note also that if $A$ is a simplicial differential graded algebra (sdga from now on), $A(K)$ also inherits in the same way an algebra structure. If $A$ and $B$ are $s d g m$ 's or $s d g a$ 's, we may define in the obvious way the tensor product $A \otimes B=$ $\oplus_{n}\left(A_{n} \otimes B_{n}\right)$ which together with the face and degeneracy operators $\partial_{i} \otimes \partial_{i}, s_{i} \otimes s_{i}$, is again a $s d g m$ or a $s d g a$. Note also that $A(K)$ is a covariant functor on $A$ and contravariant on $K$. For a topological space $X, A(X)$ shall denote $A\left(C_{*}(X)\right)$ where $C_{*}(X)$ is the simplicial set of singular simplices on $X$.

Remark that if $A$ is a $s d g m$ (resp. sdga), for any $n \geq 0$ the map $A(\Delta[n]) \rightarrow A_{n}$ defined by $\Phi \mapsto \Phi_{1_{\Delta^{n}}}$ is an isomorphism of differential graded modules (resp. differential graded algebras).

2.1. Definition. Given $q \geq 1$, a simplicial set $A$ is $q$-extendable if for any $0 \leq p<q$ and any $n \geq 0$, given $\Phi_{i} \in A_{n-1}^{p}, i=0, \ldots, n$, with $\partial_{i} \Phi_{j}=\partial_{j-1} \Phi_{i}$, for $i<j$, there exists an element $\Phi \in A_{n}^{p}$ such that $\partial_{i} \Phi=\Phi_{i}$. A is extendable if it is $q$-extendable for any $q \geq 0$.

Now, we give a technical result which allows us to conclude $q$-extendability by considering only the case $\Phi_{i}=0, i \leq n-1$.

2.2. Proposition. Assume the following: Given $\Phi \in A_{n-1}^{p}, p<q, n \geq 1$, such that $\partial_{i} \Phi=0, i<r \leq n-1$, there exists $\Psi \in A_{n}^{p}$ so that $\partial_{i} \Psi=0, i<r$, and $\partial_{r} \Psi=\Phi$.

Then, $A$ is q-extendable.

Proof. Let $\Phi_{i} \in A_{n-1}^{p}, i=0, \ldots, n$, with $\partial_{i} \Phi_{j}=\partial_{j-1} \Phi_{i}$ for $i<j$. Then consider $\Phi_{0}$ and choose $\Psi^{o} \in A_{n}^{p}$ such that $\partial_{0} \Psi^{o}=\Phi_{0}$. Define

$$
\Phi_{i}^{1}=\Phi_{i}-\partial_{i} \Psi^{o}, \quad i=0, \ldots, n,
$$

and observe that $\Phi_{0}^{1}=0$ and $\partial_{0} \Phi_{1}^{1}=0$. Apply then our hypothesis to $\Phi_{1}^{1}$ (with $r=1$ ) to find $\Psi^{1} \in A_{n}^{p}$ so that $\partial_{0} \Psi^{1}=0, \partial_{1} \Psi^{1}=\Phi_{1}^{1}$.

By the same inductive process we end up defining elements $\Phi_{i}^{j} \in A_{n-1}^{p}$ and $\Psi^{j} \in A_{n}^{p}$, $i, j=0, \ldots, n$ satisfying:

$$
\Phi_{i}^{j}=\Phi_{i}^{j-1}-\partial_{i} \Psi^{j-1}
$$




$$
\begin{aligned}
\partial_{k} \Phi_{j}^{j} & =\partial_{k} \Psi^{j}, \quad k<j, \\
\Phi_{i}^{j} & =0, \quad i<j, \quad \Phi_{j}^{j}=\partial_{j} \Psi^{j} .
\end{aligned}
$$

Finally, it is an easy calculation to show that the element $\Psi=\Psi^{o}+\cdots+\Psi^{n}$ satisfies $\partial_{i} \Psi=\Phi_{i}, 0 \leq i \leq n$.

Now and until the end of this section, we shall briefly outline how having $q$-extendability affects the development of $[3 ; \S 10]$ or $[4 ;$ chap.12]. For the next two results the proofs are as in $[3 ; \S 10]$. We just have to take care that the weaker hypothesis of $q$-extendability is enough for our purposes. We sketch the proofs for clarity.

2.3. Proposition. Let $A$ be a q-extendable sdgm and let $L \subset K$ be a subsimplicial set. Then, the induced morphisms $A^{p}(K) \rightarrow A^{p}(L)$ are surjective for any $p<q$.

Proof. Let $\Psi \in A^{p}(L)$ and assume defined $\Phi_{\sigma}, \sigma \in K_{m}, m<n$, so that it commutes with faces and degeneracies and $\Psi_{\sigma}=\Phi_{\sigma}$ for $\sigma \in L_{m}$. Next, given $\sigma \in K_{n}$ define: $\Psi_{\sigma}=\Phi_{\sigma}$ if $\sigma \in L_{n}$ and $\Phi_{\sigma}=s_{j} \Phi \tau$ if $\sigma=s_{j} \tau$; to finish, if $\sigma$ is a non-degenerate simplex of $K_{n}-L_{n}$, since $\partial_{i} \Phi_{\partial_{j} \sigma}=\partial_{j-1} \Phi_{\partial_{i} \sigma}$, for $i<j$, we make use of $q$-extendability to find $\Phi_{\sigma}$ so that $\partial_{i} \Phi_{\sigma}=\Phi_{\partial_{i} \sigma}$, for $i \leq n$.

2.4. Proposition. Let $\gamma: A \rightarrow B$ be a morphism of q-extendable sdgm such that each $\gamma_{m}: A_{m} \stackrel{\simeq}{\longrightarrow} B_{m}, m \geq 0$, induces an isomorphism in cohomology. Then, for any $p<q$ and for any simplicial set $K$

$$
H^{p}(\gamma(K)): H^{p}(A(K)) \stackrel{\cong}{\longrightarrow} H^{p}(B(K))
$$

is also an isomorphism.

Pro of. We just outline the proof briefly: The key fact here is that $\gamma(K(m), K(m-1))$ induces an isomorphism in cohomology up to degree $q$. Indeed, if each $\gamma_{m}, m \geq 0$, is a quasi-isomorphism so is $\gamma(\Delta[m]): A(\Delta[m]) \rightarrow B(\Delta[m])$ since $A(\Delta[m]) \cong A_{m}$. Hence, in view of the exact sequences,

$$
0 \rightarrow A^{p}(\Delta[m], \Delta[m-1]) \rightarrow A^{p}(\Delta[m]) \rightarrow A^{p}(\Delta[m-1]) \rightarrow 0, \quad p<q,
$$

it follows that $\gamma(\Delta[m], \Delta[m-1])$ induces isomorphism in cohomology up to degree $q$. Now we use the fact that $A(K(m), K(m-1)) \cong \Pi_{N} A(\Delta[m], \Delta[m-1])$, where $N$ denotes the set of non degenerate simplices of $K_{m}$ (see [3;lemma 10.6]), to conclude that in fact $\gamma(K(m), K(m-1))$ induces an isomorphism in cohomology up to degree $q$.

To see that $H^{p}\left(\gamma^{p}(K)\right)$ is injective, $p<q$, let $[\alpha] \in \operatorname{ker} H^{p}(\gamma(K))$ and choose $\beta \in$ $B^{p-1}$ so that $\gamma(K)(\alpha)=d \beta$. Using $q$-extendability and the above mentioned fact it is easy to construct inductively elements $\phi_{i} \in A(K, K(i-1))$ so that $\alpha-\sum_{i<m} d \phi_{i} \in$ $A(K, K(m))$. To conclude define $\phi=\sum_{i>0} \phi_{i}$. This is a well defined element in $A^{p-1}(K)$ since $\left(\phi_{i}\right)_{\sigma}=0$ if $i>|\sigma|$ and it clearly satisfies $d \phi=\alpha$, i.e., $[\alpha]=0$. The same inductive procedure shows that $H^{p}\left(\gamma^{p}(K)\right)$ is surjective.

Recall that for a given simplicial set $K$, the cochain algebra on $K$ is the differential graded algebra $C^{*}(K)=\oplus_{n \geq 0} C^{p}(K)$ in which $C^{p}(K)$ consists of maps $f: K_{p} \rightarrow R$. As usual, the "cup" product and the differential are given by

$$
(f \cup g) \sigma=(-1)^{p q} f\left(\partial_{p} \ldots \partial_{p+q} \sigma\right) g\left(\partial_{0} . \stackrel{p}{.} \partial_{0} \sigma\right), \sigma \in K_{p+q}, f \in C^{p}(K), g \in C^{q}(K),
$$




$$
(d f) \sigma=\sum_{i=0}^{p+1}(-1)^{i} f\left(\partial_{i} \sigma\right), \sigma \in K_{p+1}, f \in C^{p}(K) .
$$

On the other hand the polynomial simplicial cochain algebra is defined by $C_{P L}=$ $\oplus_{n \geq 0}\left(C_{P L}\right)_{n}$ in which $\left(C_{P L}\right)_{n}=C^{*}(\Delta[n])$. It turns out that $C_{P L}$ is extendable and for any simplical set $K$ the natural map $\eta: C_{P L}(K) \stackrel{\cong}{\longrightarrow} C^{*}(K)$, defined by $\eta(\Phi) \sigma=$ $\Phi_{\sigma}\left(1_{\Delta^{p}}\right), \sigma \in K_{p}$, is an isomorphism of differential graded algebras [3, $\left.\S 10\right]$. Hence, for a given space $X, C_{P L}(X)$ is isomorphic to the usual singular cochain complex on $X$. We shall also need the following result that again has the same proof as $[3, \S 10]$ or [4,Chap.12] but taking into account that we assume just $q$-extendability.

2.5. Proposition. If $A$ is a q-extendable sdgm then $C_{P L} \otimes A$ is also q-extendable.

3. Polynomial forms of fixed length. In this section we recall the definition of the $s d g m$ of polynomial forms of fixed length over $R$ and prove, as we stated in the introduction, the two necessary conditions to have a "de Rham theorem", i.e., Poincaré's lemma (proposition 3.1) and $q$-extendability (proposition 3.2).

Define $A_{P L}$ the simplicial differential graded algebra of polynomial forms by

$$
\left(A_{P L}\right)_{n}=\left(\Gamma\left(x_{0}, \ldots, x_{n}, d x_{1}, \ldots, d x_{n}\right), d\right)
$$

in which: each $x_{i}$ has degree 0 and $d x_{i}$ has degree $1 ; \Gamma$ denotes the divided power algebra on those generators; and $d$ is the only differential which sends $x_{i}$ to $d x_{i}, 1 \leq i \leq n$ and $d x_{0}=-d x_{1}-\cdots-d x_{n}$. Note that $\left(A_{P L}\right)_{n}=\Gamma\left(x_{0}, \ldots, x_{n}, d x_{0}, \ldots, d x_{n}\right) / \sum_{i} d x_{i}$. The face and degeneracy operators are the unique algebra morphisms defined as follows:

$$
\begin{gathered}
\partial_{i}: A_{P L_{n+1}} \longrightarrow A_{P L_{n},} \quad 0 \leq i \leq n+1, \\
\partial_{i} \gamma^{\alpha}\left(x_{r}\right)=\left\{\begin{array}{ll}
\gamma^{\alpha}\left(x_{r}\right), & r<i, \\
0, & r=i, \\
\gamma^{\alpha}\left(x_{r-1}\right), & r>i,
\end{array} \quad \partial_{i} d x_{r}= \begin{cases}d x_{r}, & r<i, \\
0, & r=i, \\
d x_{r-1}, & r>i,\end{cases} \right. \\
s_{j}: A_{P L_{n}} \longrightarrow A_{P L_{n+1},} \gamma^{\alpha}\left(x_{r}\right)=\left\{\begin{array}{ll}
\gamma^{\alpha}\left(x_{r}\right), & 0 \leq j \leq n, \\
\sum_{p+q=\alpha} \gamma^{p}\left(x_{j}\right) \gamma^{q}\left(x_{j+1}\right), & r=j, \\
\gamma^{\alpha}\left(x_{r+1}\right), & r>j,
\end{array} \quad s_{j} d x_{r}= \begin{cases}d x_{r}, \\
d x_{j}+d x_{j+1}, & r=j, \\
d x_{r+1}, & r>j .\end{cases} \right.
\end{gathered}
$$

Observe that $\left(A_{P L}\right)_{n}=\oplus_{p, q \geq 0}\left(A_{P L}\right)_{n}^{p, q}$ is bigraded by the total degree and by the "wordlength" degree: an element of $\left(A_{P L}\right)_{n}^{p, q}$ is a linear combination of terms of the form

$$
\gamma^{i_{0}}\left(x_{0}\right) \ldots \gamma^{i_{n}}\left(x_{n}\right) d x_{j_{1}} \ldots d x_{j_{p}}, \quad i_{k} \geq 0,1 \leq j_{1}<\ldots<j_{p} \leq n, i_{0}+\cdots+i_{n}+p=q .
$$

Clearly, $\left(A_{P L}\right)_{n}^{p, q}=0$ if either $p>n$ or $p>q$. On the other hand the operators $\partial_{i}$ and $s_{i}$ preserve both degrees so we may define, for any integer $q \geq 0$, the $\operatorname{sdgm} A_{P L}^{*, q}$ of polynomial forms of length $q$ by

$$
\left(A_{P L}^{*, q}\right)_{n}=\oplus_{p \geq 0}\left(A_{P L}\right)_{n}^{p, q} .
$$

From now on, for simplicity of notation, we shall write $A$ and $A^{*, q}$ instead of $A_{P L}$ and $A_{P L}^{*, q}$.

3.1. Proposition. $H^{*}\left(A_{n}^{*, q}\right)=H^{0}\left(A_{n}^{*, q}\right) \cong R$. 
Proof. The morphism

$$
\left(\Gamma\left(x_{0}\right), 0\right) \otimes \Gamma\left(x_{1}, \ldots, x_{n}, d x_{1}, \ldots, d x_{n}\right) \rightarrow A_{n}
$$

given by

$$
\varphi\left(\gamma^{m}\left(x_{0}\right) \otimes \Phi\right)=\Phi \cdot \sum_{a_{0}+\cdots+a_{n}=m} \gamma^{a_{0}}\left(x_{0}\right) \ldots \gamma^{a_{n}}\left(x_{n}\right)
$$

is an isomorphism of differential graded algebras. Indeed a calculation shows that it preserves the product. To see that it commutes with the differential it is enough to see that, in $A_{n}, d\left(\sum_{a_{0}+\cdots+a_{n}=m} \gamma^{a_{0}}\left(x_{0}\right) \ldots \gamma^{a_{n}}\left(x_{n}\right)\right)=0$. For that write,

$$
\sum_{a_{0}+\cdots+a_{n}=m} \gamma^{a_{0}}\left(x_{0}\right) \ldots \gamma^{a_{n}}\left(x_{n}\right)=\gamma^{m}\left(x_{0}\right)+\gamma^{m-1}\left(x_{0}\right) C_{1}+\cdots+\gamma\left(x_{0}\right) C_{m-1}+C_{m},
$$

and a straightforward computation shows that

$$
d\left(\gamma^{i}\left(x_{0}\right)\right) C_{m-i}=-\gamma^{i-1}\left(x_{0}\right) d C_{m-i+1}, \quad 0 \leq i \leq m \quad\left(\text { here } C_{0}=1\right) .
$$

On the other hand, filter both sides by the differential modules

$$
\Gamma^{\leq p}\left(x_{0}\right) \otimes \Gamma\left(x_{1}, \ldots, x_{n}, d x_{1}, \ldots, d x_{n}\right) .
$$

Note that $\varphi$ respects the filtration and the induced map in the associated graded module is the identity. Hence $\varphi$ is an isomorphism and thus, since

$$
\Gamma\left(x_{1}, \ldots, x_{n}, d x_{1}, \ldots, d x_{n}\right)
$$

is contractible, $H^{*}\left(A_{n}\right)=H^{0}\left(A_{n}\right) \cong \Gamma\left(x_{0}\right)$. Therefore,

$$
H^{*}\left(A_{n}^{*, q}\right)=H^{0}\left(A_{n}^{*, q}\right) \cong R \cdot \gamma^{q}\left(x_{0}\right) \cong R
$$

3.2. Proposition. $A^{*, q}$ is q-extendable.

Proof. We introduce the following notation: for any $J=\left(j_{0}, \ldots, j_{n}\right) \in \mathbb{N}^{n+1}$ let $|J|=j_{0}+\cdots+j_{n}$ and $\gamma^{J}\left(x_{J}\right)=\gamma^{j_{0}}\left(x_{0}\right) \ldots \gamma^{j_{n}}\left(x_{n}\right)$. Then observe that the elements $\left\{\gamma^{J}\left(x_{J}\right),|J|=p\right\}$ form a basis for $\oplus_{k \geq 0} A_{n}^{k, p+k}$ as a module over $\Gamma\left(d x_{1}, \ldots, d x_{n}\right)$.

In view of proposition 2.2 it is enough to consider $\Psi \in A_{n-1}^{p, q}$ with $p<q$, such that $\partial_{i} \Psi=0,0 \leq i \leq n-1$ and produce and element $\Phi \in A_{n}^{p, q}$ so that $\partial_{i} \Phi=0,0 \leq i \leq n-1$, $\partial_{n} \Phi=\Psi$. By the observation above write

$$
\Psi=\sum_{|J|=q-p} \gamma^{J}\left(x_{J}\right) w_{J}, \quad w_{J} \in \Gamma^{p}\left(d x_{1}, \ldots, d x_{n-1}\right), \quad J=\left(j_{0}, \ldots, j_{n-1}\right) .
$$

Now for $0 \leq i \leq n-1,0=\partial_{i} \Psi=\sum_{|J|=q-p} \partial_{i} \gamma^{J}\left(x_{J}\right) \partial_{i} w_{J}$. But note that

$$
\partial_{i} \gamma^{J}\left(x_{J}\right)=\left\{\begin{array}{ll}
0 & \text { if } \quad j_{i}>0, \\
\gamma^{J^{\prime}}\left(x_{J^{\prime}}\right) & \text { if } \quad j_{i}=0
\end{array} \text { with } \quad J^{\prime}=\left(j_{0}, \ldots, j_{i-1}, j_{i+1}, \ldots, j_{n-1}\right),\right.
$$

in which the elements $\gamma^{J^{\prime}}\left(x_{J^{\prime}}\right)$ form again a basis of $\oplus_{k \geq 0} A_{n-2}^{k, q-p+k}$ as a module over $\Gamma\left(d x_{1}, \ldots, d x_{n-2}\right)$. Hence

$$
\partial_{i} \Psi=0 \Longrightarrow\left\{\begin{array}{lll}
\partial_{i} \gamma^{J}\left(x_{J}\right)=0 & \text { if } & j_{i}>0 \\
\partial_{i} w_{J}=0 & \text { if } & j_{i}=0
\end{array}\right.
$$


Next consider those $i_{1}, \ldots, i_{r} \in\{0, \ldots, n-1\}$ for which $j_{i_{1}}=\cdots=j_{i_{r}}=0$. The fact that $\partial_{i_{k}} w_{J}=0, k=1, \ldots, r$ lets us write

$$
w_{J}=d x_{i_{1}} \ldots d x_{i_{r}} \cdot w^{\prime} .
$$

Observe that for this it is essential that $p<q$ : Indeed since $p<q$ there is at least one $j_{i}>0$ and therefore $r \leq n-1$ which is necessary for $(*)$ to hold. Otherwise it is not true since in $A_{n-1}, d x_{0} \ldots d x_{n-1} \cdot w^{\prime}$ is always zero.

Finally, consider the element $\widetilde{w}_{J} \in A_{n}$, defined by $\widetilde{w}_{J}=w_{J}$ (the only possible difference lies in the fact that in $A_{n}, d x_{0}=-\sum_{i=1}^{n} d x_{i}$ while in $A_{n-1}, d x_{0}=-\sum_{i=1}^{n-1} d x_{i}$ ) and observe that

$$
\begin{aligned}
\partial_{i}\left(\gamma^{J}\left(x_{J}\right) \widetilde{w}_{J}\right) & =\partial_{i} \gamma^{J}\left(x_{J}\right) \partial_{i} w_{J}=0, \quad i<n, \\
\partial_{n}\left(\gamma^{J}\left(x_{J}\right) \widetilde{w}_{J}\right) & =\gamma^{J}\left(x_{J}\right) w_{J} .
\end{aligned}
$$

Therefore, the element

$$
\Phi \in A_{n}, \quad \Phi=\sum_{|J|=q-p} \gamma^{J}\left(x_{J}\right) \widetilde{w}_{J}
$$

satisfies $\partial_{i} \Phi=0, i<n, \partial_{n} \Phi=\Psi$, and the proposition follows.

3.3. REMARK. (i) This is the best possible result since $A^{*, q}$ is not $q+1$-extendable: consider in $A_{q}^{q, q}$ the volume form $\Psi=d x_{1} \ldots d x_{q}$ which is obviously non-zero and satisfies $\partial_{i} \Psi=0,0 \leq i \leq q$. We claim that there is no $\Phi \in A_{q+1}^{q, q}$ with $\partial_{i} \Phi=0,0 \leq i \leq q$, $\partial_{q+1} \Phi=\Psi$. For it is easy to see that the first condition forces $\Phi=0$.

(ii) Also observe that the standard proof of extendability of $A_{P L}$ over the rationals due originally to Sullivan [8] (see also [3] or [4]) cannot be applied or modified in our context since the "weight" of the forms is fixed.

4. The main results. Consider the simplicial morphisms of $s d g m$

$$
\alpha_{m}: C_{P L m} \longrightarrow C_{P L m} \otimes A_{m}^{*, q}, \quad \beta_{m}: A_{m}^{*, q} \longrightarrow C_{P L m} \otimes A_{m}^{*, q}, \quad m \geq 0,
$$

defined respectively by $\alpha_{m}(\Phi)=\Phi \otimes \gamma^{q}\left(x_{0}\right), \beta_{m}(\Psi)=1 \otimes \Psi$. These maps determine, for any simplicial set $K$, morphisms of differential graded modules

$$
\alpha(K): C_{P L}(K) \longrightarrow\left(C_{P L} \otimes A^{*, q}\right)(K) \longleftarrow A^{*, q}(K): \beta(K)
$$

Then, we have

4.1. TheOREm. For any $p<q, H^{p}(\alpha(K))$ and $H^{p}(\beta(K))$ are isomorphisms.

P r o of. By prop. 3.1, $H^{*}\left(A_{n}^{*, q}\right)=H^{0}\left(A_{n}^{*, q}\right) \cong R$. On the other hand, as in the classical case, one sees that $H^{*}\left(C_{P L_{n}}\right)=H^{0}\left(C_{P L_{n}}\right) \cong R$ and therefore, applying the Künneth theorem $H^{*}\left(C_{P L} \otimes A^{*, q}\right) \cong R$. Thus it is immediate that the morphisms induced in cohomology by $\alpha_{m}$ and $\beta_{m}$ are isomorphisms. To finish recall that $C_{P L}$ is extendable while, in view of propositions 2.5 and $3.2, A^{*, q}$ and $C_{P L} \otimes A^{*, q}$ are $q$-extendable. Hence the theorem follows applying proposition 2.4 .

For a given element of $A_{p}^{p, q}$ of the form

$$
\psi=\lambda \gamma^{i_{0}}\left(x_{0}\right) \ldots \gamma^{i_{p}}\left(x_{p}\right) d x_{1} \ldots d x_{p}, \quad i_{0}+\cdots+i_{p}+p=q,
$$

we define the scalar 


$$
q ! \int_{\Delta_{p}} \psi=\lambda q ! \int_{\Delta_{p}} \frac{x_{0}^{i_{0}} \ldots x_{p}^{i_{p}}}{i_{0} ! \ldots i_{p} !} d x_{1} \ldots d x_{p},
$$

which is, after an easy calculation, nothing but $\lambda$ (for $p=0$ we set $q$ ! $\int \lambda \gamma^{q}\left(x_{0}\right)=\lambda$ ). We extend linearly this to $A_{p}^{p, q}$ and in this way we may define the morphism of differential graded modules

$$
q ! \int: A^{*, q}(K) \longrightarrow C^{*}(K)
$$

by $\left(q ! \int \Phi\right)(\sigma)=q ! \int_{\Delta^{p}} \Phi_{\sigma}, \sigma \in K_{p}$. Indeed this is a well defined morphism, and the fact that it commutes with the differential is an immediate consequence of the Stokes's Theorem. Then, our main result reads:

4.2. TheOrem. For any simplicial set $K$,

$$
q ! \int: A^{*, q}(K) \stackrel{\simeq}{\longrightarrow} C^{*}(K)
$$

induces an isomorphism in cohomology in degree $p<q$.

Pro of. As in [4, Chap.14] we prove that for any $p<q, H^{p}\left(q ! \int\right)=H^{p}\left(\alpha^{-1}\right) \circ H^{p}(\beta)$. For that consider the maps $\gamma_{m}: C_{P L m} \otimes A_{m}^{*, q} \rightarrow C_{P L m}$ defined by $\gamma_{m}(\Phi \otimes \Psi)=\left(q ! \int \Psi\right) \Phi$ (here we have identified $A_{m}^{*, q}$ with $A^{*, q}(\Delta[m])$. Then $\gamma_{m}$ commutes with the differential, face and degeneracy operators, and therefore it induces a map

$$
\gamma(K):\left(C_{P L} \otimes A^{*, q}\right)(K) \longrightarrow C_{P L}(K) \cong C^{*}(K)
$$

which satisfies $\gamma \alpha=1$ and $\gamma \beta=q ! \int$. Hence $H^{p}\left(q ! \int\right)=H^{p}(\alpha)^{-1} \circ H^{p}(\beta)$ and the theorem follows.

In particular, for a given topological space $X$ we have

4.3. THEOREM.

$$
q ! \int: A^{*, q}(X) \stackrel{\simeq}{\longrightarrow} C^{*}(X)
$$

induces an isomorphism in cohomology up to degree $q$.

\section{References}

[1] H. Cartan, Théories cohomologiques, Invent. Math. 35 (1976), 261-271.

[2] B. Cenkl, Cohomology operations from higher products in the de Rham complex, Pacific Journal of Math. 1401 (1989), 21-33.

[3] Y. Félix, S. Halperin and J. C. Tomas, Rational Homotopy Theory, Preprint Univ. of Toronto, version 96.2, (1996).

[4] S. Halperin, Lectures on minimal models, Mémoire de la Soc. Math. de France, 9/10 (1983).

[5] P. May, Simplicial objects in algebraic topology, Van Nostrand, 1967.

[6] E. Y. Miller, De Rham cohomology with arbitrary coefficients, Topology 17 (1978), 193203.

[7] D. Quillen, Rational homotopy theory, Annals of Math. 90 (1969), 205-295.

[8] D. Sullivan, Infinitesimal Computations in Topology, Publ. de l'I.H.E.S. 47 (1978), 269331.

[9] R. Swan, Thom's theory of differential forms on simplicial sets, Topology 14 (1975). 271273. 